\title{
Donald Daniel Leslie, Daye Yang, Ahmed Youssef (éds.), Islam in Traditional China. A Bibliographical Guide
}

Sankt Augustin - Nettetal, Institut Monumenta Serica - Steyler Verlag, coll. « Monumenta Serica Monograph Series », LIV, 2006, 398 p.

\section{Françoise Aubin}

\section{(2) OpenEdition}

\section{Journals}

Édition électronique

URL : http://journals.openedition.org/assr/6622

DOI : $10.4000 /$ assr.6622

ISSN : $1777-5825$

Éditeur

Éditions de l'EHESS

Édition imprimée

Date de publication : 1 juin 2007

Pagination : $97-251$

ISBN : 978-2-7132-2143-9

ISSN : 0335-5985

\section{Référence électronique}

Françoise Aubin, «Donald Daniel Leslie, Daye Yang, Ahmed Youssef (éds.), Islam in Traditional China. A

Bibliographical Guide ", Archives de sciences sociales des religions [En ligne], 138 | avril - juin 2007

document 138-58, mis en ligne le 12 septembre 2007, consulté le 21 septembre 2020. URL : http://

journals.openedition.org/assr/6622 ; DOI : https://doi.org/10.4000/assr.6622

Ce document a été généré automatiquement le 21 septembre 2020.

(C) Archives de sciences sociales des religions 


\section{Donald Daniel Leslie, Daye Yang, Ahmed Youssef (éds.), Islam in Traditional China. A Bibliographical Guide}

Sankt Augustin - Nettetal, Institut Monumenta Serica - Steyler Verlag, coll. « Monumenta Serica Monograph Series », LIV, 2006, 398 p.

\section{Françoise Aubin}

1 Le sinologue australien Donald D. Leslie a été, dans les années 1980, le pionnier d'une découverte sérieuse de l'islam en langue chinoise dans les textes; et ses deux ouvrages, qui ont tracé la voie, sont restés les piliers fondamentaux de toute étude sur la question. Ce fut d'abord une bibliographie des sources jusqu'à la fin du XviII ${ }^{\mathrm{e}}$ siècle : Islamic Literature in Chinese, Late Ming and Early Ch'ing (Canberra, The Canberra College of Advanced Education, 1981), puis un récit historique s'arrêtant aussi à l'aube du XIx ${ }^{\mathrm{e}}$ siècle : Islam in Traditional China : A Short History to 1800 (même éditeur, 1986). Le présent travail, qui va être désormais l'ouvrage obligatoire de référence, est autre chose qu'une mise à jour de la première bibliographie. En témoigne d'ailleurs la taille respective des deux volumes : moins de deux cents pages pour le premier, presque quatre cents pour le second, et la collaboration des deux collègues que l'auteur principal avait déjà associés à plusieurs de ses publications qui servent ici de trame.

2 Que nous apporte donc de nouveau et d'indispensable à connaître ce guide bibliographique? On ne peut mieux en juger qu'en examinant dans l'ordre le contenu de chaque chapitre, sans s'amuser au jeu d'éventuelles omissions ou fautes de frappe, absurde face à une telle somme. Pour commencer, une chronologie des principaux événements politiques et littéraires, qui ont scandé l'existence des communautés musulmanes de Chine, nous donne les points de repère toujours vivants dans la mémoire collective (pp. 19-22). Trois brefs glossaires enseignent au sinologue le sens des termes chinois et arabo-persans les plus courants dans les titres des ouvrages qui vont être cités (pp. 27-30) - pour une plus ample information, on peut maintenant se 
reporter, en outre, à A Glossary of Chinese Islamic Terms de Wang Jianping (Richmond, Curzon, 2001 [et non 2002 comme il est écrit p. 30], 196 p.), que D. D. Leslie a marqué de deux astérisques, et non de trois, sans doute en raison d'un certain nombre d'insuffisances. Les titres parmi les plus marquants sont en effet évalués par un à trois, et plus rarement quatre, astérisques, afin de permettre à l'utilisateur de faire rapidement son choix (il faut mettre en garde le lecteur qu'un titre rendu alléchant par trois astérisques, p. 126, The Legacy of Islam in China: An International Symposium in Memory of Josef Fletcher, Harvard, 1989, n'a en fait jamais été publié).

3 La première liste bibliographique (pp. 35-48) concerne les répertoires des sources primaires et des études ainsi que des catalogues de bibliothèques et de fonds d'archives. Puis on entre dans le vif du sujet avec un inventaire des sources: les inscriptions musulmanes en arabe puis en chinois (pp. 51-61) ; les registres familiaux ou jiapu (pp. 76-79) ; les œuvres littéraires en chinois léguées par les grands 'âlim du passé (pp. 62-70) et leurs sources arabes et persanes identifiées - on ne comprend pas très bien pourquoi l'inventaire des sources moyen-orientales se trouve scindé en deux listes (pp. 70-75 et 85-104, cette dernière incluant les traductions et études occidentales qui en ont été données) ; des sources chinoises non religieuses (pp. 79-84).

4 La liste suivante, formant la troisième partie (pp. 111-132), qui reprend certains des matériaux mentionnés précédemment, recense, sous forme abrégée, quantité de bibliographies et de catalogues, ainsi que les périodiques les plus utiles, les thèses et travaux inédits. La quatrième partie (pp. 135-186) répartit les titres cette fois par thèmes traités: toponymes et anthroponymes, démographie, apports scientifiques, chronologie dynastique, sociologie et, bien sûr, religion, mais fort brièvement (pp. 182-184).

5 La cinquième partie, la plus copieuse (pp. 189-326), forme le cœur de l'ouvrage, avec une belle bibliographie des études sur le sujet, très clairement présentée, par noms d'auteurs en langues occidentales puis en chinois et en japonais, incluant le plus souvent l'essentiel de la table des matières des livres cités. À côté des vieux titres classiques, les années 1990 sont largement représentées. Malheureusement, les travaux publiés entre 1999 et 2004 (ainsi que quelques-uns plus anciens qui avaient été oubliés) forment l'objet d'addenda (pp. 377-398) qui ne sont pas pris en compte par les index de la partie principale - un index des titres chinois et japonais et un index des noms propres et des matières en chinois, en japonais, en arabe et en langues occidentales. De telle sorte qu'un utilisateur pressé peut fort bien passer à côté de toutes les publications récentes. Un des mérites de l'ouvrage est d'inclure l'ensemble de la production française en islamologie chinoise. Outre des titres en allemand, néerlandais, italien, espagnol, arabe, quelques-uns sont aussi mentionnés en indonésien et en urdu. Mais les auteurs russes, eux, n'apparaissent que pour leurs articles traduits ou écrits directement en anglais. La bibliographie chinoise est bien pourvue en caractères chinois ; malencontreusement, il n'en est pas de même pour la partie japonaise, qui ne bénéficie d'aucun kanji, ni pour les noms propres, ni pour les titres. Les étudiants entrant dans le sujet apprécieront de trouver, en outre, dans ce riche manuel quelques titres d'ouvrages classiques lointainement liés au thème principal, ainsi ceux traitant du Xinjiang ou des minorités en RPC (pp. 251-254 par exemple); mais il leur faudra de l'obstination pour ne pas se laisser décourager par la complexité du système des renvois. 
6 À côté de ce volume que le directeur de l'institut Monumenta Serica, le P. Roman Malek, a édité avec son soin habituel, les deux premières publications de D. D. Leslie citées ci-dessus font piètre mine, tapées à la machine, les caractères chinois écrits à la main. Leur contenu est-il aussi obsolète que l'est leur présentation extérieure? Nullement! Islamic Literature in Chinese continuera à être utilisé pour son inventaire raisonné des œuvres de l'islam littéraire chinois des $\mathrm{XVII}^{\mathrm{e}}$ et $\mathrm{xvIII}{ }^{\mathrm{e}}$ siècles et pour les abrégés bibliographiques des auteurs. Même les encyclopédies chinoises d'islamologie qui fournissent, elles aussi, des notices sur les œuvres et les auteurs ne supplantent pas le compendium de D. D. Leslie et ses détails qu'on ne saurait dénicher autre part, ainsi la liste des éditions successives de chaque titre et les bibliothèques à travers le monde où l'on peut les trouver hors de Chine. Et bien que l'histoire des communautés musulmanes de Chine ait progressé de-ci de-là pour une époque particulière, surtout le $\mathrm{XIX}^{\mathrm{e}}$ siècle, Islam in Traditional China demeure la seule histoire digne de confiance, continue jusqu'à la fin du xviII ${ }^{\mathrm{e}}$ siècle et bâtie sur les sources primaires. Quant au présent travail, même s'il mentionne les œuvres originales, l'essentiel de son contenu est une bibliographie des études en toutes langues sur ces textes et sur les hommes individus marquants ou groupes en communautés. Désormais, l'islamologie chinoise est entrée dans le champ de la connaissance accessible à tous, et nul ne peut plus ignorer que le domaine est vaste, déjà bien labouré, mais encore ouvert à de multiples questions. Toutefois, nous conseillerons à l'amateur de commencer sa lecture par les addenda procurant les titres les plus récents. 